Open Peer Review on Qeios

\title{
The potential role of Monocyte Distribution Width (MDW) as early post-operative sepsis biomarker
}

\author{
Davide Baiamonte, Silvia Altomare, Rosa Giaimo, Marco VELLA ${ }^{1}$, Piero Mannone ${ }^{1}$, Pinelli Mirko, Gabriele \\ Tulone, Luisa AGNELLO, Matteo Vidali, Bruna Lo Sasso, Rosaria Vincenza Giglio ${ }^{1}$, Caterina Maria Gambino, \\ Nicola Pavan, Marcello Ciaccio $^{1}$, Alchiede Simonato ${ }^{1}$
}

1 University of Palermo

Funding: The author(s) received no specific funding for this work.

Potential competing interests: The author(s) declared that no potential competing interests exist.

\section{Abstract}

Sepsis is one of the most dangerous post-operative complications. Recently, Monocyte Distribution Width (MDW), a blood test parameter able to measure morphological changes in monocytes, has been proposed and investigated as a new promising biomarker of sepsis. The aim of our study was to evaluate if MDW can be influenced by surgery to better understand if it could be use as a sepsis marker. All clinical and laboratories data were collected of all patients admitted from April to August 2021 to the Unit of Urology of our Department for elective kidney and ureteral stones surgery. The blood samples collected to evaluate MDW of each patient were analyzed on the UniCel DxH 900 analyzer (Beckman Coulter, Inc., USA), with an MDW value of 23 considered as significative value. Times of collection of blood samples were before surgery (BS), after surgery (AS) and at discharge time (DT) In all 66 patients enrolled in this study, while White Blood Cells (WBC) significantly increased after surgery (BS:7.95x109/L vs AS:11.4x109/L; $p<0.001$ ) with a trend to normalization at discharge (AS:11.40x109/L vs DT:9.90x109/L; p:0.021), there were no statistically significative differences for MDW (BS:17.6 vs AS:18.1; $\mathrm{p}: 1$ ) before and after surgery, as well comparing before surgery and at the time of discharge (BS:17.6 vs DT:18.5; p:0.237). In conclusion, MDW is not affected by surgical procedures and is easily measurable when compared to other biomarkers, considering that is part of a routine CBC. For these reasons, MDW could be a valid, cost-effective and fast biomarker to predict the risk of post-operative sepsis in elective surgery

\section{Introduction:}

Sepsis is one of the most dangerous post-operative complications, responsible of several morbidity and mortality. Surgery causes physiological diffuse inflammatory response in the organism that alters by itself the most routinely used sepsis biomarkers, like Pro-calcitonin and C-reactive protein. Recently, Monocyte Distribution Width (MDW), a blood test parameter able to measure morphological changes in monocytes, has been proposed and investigated as a new promising biomarker of sepsis. The aim of this study was to 
evaluate if MDW can be influenced by surgery.

\section{Methods:}

In our observational retrospective study, all patients admitted from April to August 2021 to the Unit of Urology of our Department for elective kidney and ureteral stones surgery were enrolled. All patients clinical and laboratories data were collected. Complete blood count (CBC) and MDW were assessed before surgery (BS), the day after the procedure (AS) and at patients discharge time (DT). Exclusion criteria were SIRS/sepsis at admission time or during hospitalization, neutropenia, recent chemotherapy or immunosuppressant therapy, immunodeficiency syndromes, hematological disorders and failure to determine the MDW parameter. The blood samples collected to evaluate MDW of each patient were analyzed on the UniCel DxH 900 analyzer (Beckman Coulter, Inc., USA). A cut-off of 23 was used to determine MDW significative value. Quantitative variables were reported with median and interquartile range, while qualitative variables with frequencies. Differences between paired groups for continuous variables were estimated by Wilcoxon Test with Bonferroni's correction.

\section{Results:}

66 patients were enrolled in this study, with a median age of 57 years (IQR 48-64y.o.). WBC significantly increased after surgery (BS:7.95×109/L vs AS:11.4×109/L; $p<0.001$ ) with a trend to normalization at discharge (AS:11.40x109/L vs DT:9.90x109/L; p:0.021). Same tendency was observed with neutrophiles (BS:5.10x109/L vs AS:8.70x109/L; $p<0.001$ and AS:8.70x109/L vs DT:6.40x109/L; p:0.012). Lymphocytes usually decreased immediately after surgery (BS:1.89x109/L vs AS:1.40x109/L; p:0.012). There were no statistically significative differences for monocytes (BS:0.57x109/L vs AS:0.70x109/L; p:0.714) and MDW (BS:17.6 vs AS:18.1; p:1) before and after surgery, as well comparing before surgery and at the time of discharge (respectively BS:0.57x109/L vs DT:0.70x109/L; p:0.225 and BS:17.6 vs DT:18.5; p:0.237).

\section{Conclusions:}

In this study we found that MDW is not affected by surgical procedures. Moreover, MDW is easily measurable when compared to other biomarkers, considering that is part of a routine $\mathrm{CBC}$. For these reasons, MDW could be a valid, cost-effective and fast biomarker to predict the risk of post-operative sepsis in elective surgery, improving post-operative patients' management. 